\title{
Radioactive Elements of Low Atomic Number
}

\author{
W. A. Fowler, L. A. Delsasso and C. C. Lauritsen, The W. K. Kellogg Radiation Laboratory, \\ California Institute of Technology
}

(Received February 21, 1936)

Radioactive elements of low atomic number have been produced by the transmutation of various elements from lithium to fluorine by high velocity deuterons. The distribution in energy of the electrons and positrons emitted by these elements has been determined by employing a Wilson cloud chamber traversed by a magnetic field. The electron spectra have been found to have maximum energies from 5 to $13 \mathrm{MEV}$ while the positron spectra have maximum energies from 1 to $2 \mathrm{MEV}$. The form of the spectra has been found to be in agreement with a modification of the Fermi theory of beta-decay proposed by Konopinski and Uhlenbeck. The correlation of the maximum energies of the spectra $w$ ith the energies calculated from the reactions in which the radioactive element is involved is shown to await a more accurate determination of the difference in mass between the neutron and proton.
GINCE the discovery of artificial radioactivity $\boldsymbol{D}$ by Curie and Joliot ${ }^{1}$ in 1934 investigations have been carried out in many laboratories to determine the properties of the emitted radiation and the nature of the emitting substances. In this laboratory it has been possible to produce various radioactive elements of low atomic number by bombarding certain of the elements from lithium to fluorine with high velocity deuterons. It is the purpose of this paper to discuss the determination of the half-lives of these elements and the distribution in energy of the particles which they emit. In addition data will be presented concerning the mass-energy balances of the transmutations in which these elements are involved.

Among the unstable elements both electron and positron emitters have been found. All of the electron emitters of low atomic numbers are believed to have atomic weights $A$ given in terms of their nuclear charge $Z$ by $A=2 Z+2$. The elements bombarded in order to produce the unstable elements consist in general of two isotopes of atomic weights given by $A=2 Z$ or $A=2 Z+1$, and it is the transmutation of the heavier of these which is believed to lead to the production of the radioactive nucleus. The reaction can be written in general as

$$
\mathrm{I}(a){ }_{z} X^{2 z+1}+{ }_{1} \mathrm{H}^{2} \rightarrow{ }_{z} X^{2 z+2}+{ }_{1} \mathrm{H}^{1}+Q_{1},
$$

where $Q_{1}$ is the amount of energy released in the reaction. Present theories of the beta-decay postulate the emission of a neutrino $\nu$ simultaneously with the disintegration electron $e^{-}$.

\footnotetext{
${ }^{1}$ Curie and Joliot, Comptes rendus 198, 254 (1934).
}

In order to complete the electronic structure of the stable atom resulting from the disintegration an extranuclear orbital electron $e_{0}^{-}$must be added. Hence, when written in the form in which atomic masses may be employed, the equation representing the radioactive disintegration is

$$
\mathrm{I}(b) \quad{ }_{z} X^{2 z+2} \rightarrow_{z+1} Y^{2 z+2}+e^{-}-e_{0}^{-}+\nu+Q_{2},
$$

where ${ }_{z+1} Y^{2 z+2}$ is a stable isotope of the element of next higher atomic number. The same isotope is produced in the reaction

$$
\mathrm{I}(c) \quad{ }_{2} X^{2 z+1}+{ }_{1} \mathrm{H}^{2} \rightarrow{ }_{z+1} Y^{2 z+2}+{ }_{0} n^{1}+Q_{3} .
$$

It will be of some interest to compare the energy released in this transmutation with the total energy change in the first two reactions. This comparison is accomplished by combining the above three equations into the form

$$
\mathrm{I}(d) \quad Q_{1}+Q_{2}-Q_{3}=\left({ }_{0} n^{1}-{ }_{1} \mathrm{H}^{1}\right)-\nu \bumpeq 0.5 \mathrm{MEV} .
$$

The positron emitters of low atomic numbers have atomic weights given by $A=2 Z-1$ and when produced by deuteron bombardment result from the transmutation of the lighter isotope of the element of next lower atomic number, the reaction being

$$
\mathrm{II}(a) \quad{ }_{z} X^{2 z}+{ }_{1} \mathrm{H}^{2} \rightarrow{ }_{z+1} Y^{2 z+1}+{ }_{0} n^{1}+T_{1} .
$$

The radioactive disintegration with the emission of a positron $e^{+}$is represented by

$$
\text { II (b) } \quad{ }_{z+1} Y^{2 z+1} \rightarrow{ }_{z} X^{2 z+1}+e^{+}+e_{0}{ }^{-}+\nu+T_{2}
$$

and the reaction which leads directly to the final stable nucleus is

$$
\mathrm{II}(c) \quad{ }_{z} X^{2 z}+{ }_{1} \mathrm{H}^{2} \rightarrow{ }_{z} X^{2 z+1}+{ }_{1} \mathrm{H}^{1}+T_{3} .
$$


The above three equations can be reduced to the form

$$
\mathrm{II}(d) \quad \begin{aligned}
T_{1}+T_{2}-T_{3}= & \left({ }_{1} \mathrm{H}^{1}-{ }_{0} n^{1}\right) \\
& -e^{+}-e_{0}{ }^{-}-\nu \simeq-1.5 \mathrm{MEV} .
\end{aligned}
$$

\section{Experimental Technique}

The high voltage equipment employed for the acceleration of the bombarding deuterons has been described previously. ${ }^{2}$ In this series of experiments peak voltages from 600 to $1000 \mathrm{kv}$ and currents of the order of one microampere to targets three millimeters in diameter were employed.

Targets of the elements to be disintegrated were placed at an angle of $45^{\circ}$ with the incident beam in a cylindrical holder projecting into a horizontal Wilson cloud chamber as shown in Fig. 1. The electrons or positrons entered the chamber through a thin copper foil soldered over a window cut in the holder opposite the target. A quartz ring, visible through a glass section of the ion tube, fluoresced under bombardment and made it possible to direct the beam on to the

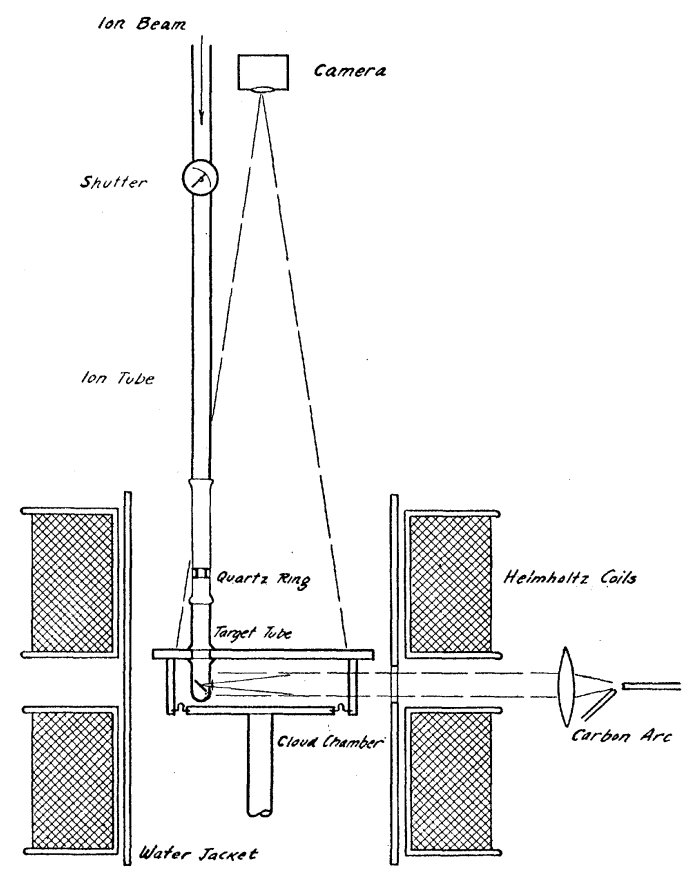

FIG. 1. Arrangement of the target assembly, cloud chamber and Helmholtz coils used in the determination of electron and positron distributions in energy.

${ }^{2}$ Crane, Lauritsen and Soltan, Phys. Rev. 45, 507 (1934). target. Movement of the chamber necessary to line up the beam was permitted by a sylphon in the ion tube. In studying radioactive effects of half-lives greater than one-tenth second, a magnetically operated shutter in the ion tube was closed and the anode voltage of the ion source cut off during the chamber expansion so that only delayed effects were observed.

The chamber proper in which tracks were produced measured $15 \mathrm{~cm}$ in diameter and $4 \mathrm{~cm}$ in depth. A flexible rubber diaphragm formed the seal between the piston and the chamber wall. Compression of the chamber was accomplished by applying compressed air to a sylphon connected to the piston by a drive shaft. An adjustable expansion ratio stop and a dash pot were located between the piston and the operating sylphon. The chamber was operated on a cycle of approximately 16 seconds, the timing of all the necessary equipment being electrically controlled from a motor-driven contact system.

The magnetic field in which the paths of the electrons or positrons were bent in order to make energy determinations possible was provided by a pair of Helmholtz coils concentric with the chamber and at equal distances above and below it. The magnetic field was measured with a fluxmeter and searchcoil calibrated in an accurately known field. Calculations from the dimensions of the coils yielded values five percent lower than the measured values, the discrepancy being certainly due to the imperfectly known geometry of the coils. The field was found to vary less than one percent across the face of the chamber.

Photographs were taken normal to the plane of the chamber. These photographs were reprojected through the same optical system to actual size and the curvature of the tracks measured by visual comparison with arcs of known radii of curvature drawn on heavy white paper. The successive arcs differed in radius by one-half centimeter. A typical cloud chamber photograph is shown in Fig. 2.

\section{Errors of Measurement}

The determination of the distribution in energy of the electrons and positrons is subject to several errors which it is well to enumerate before considering the results obtained for the 


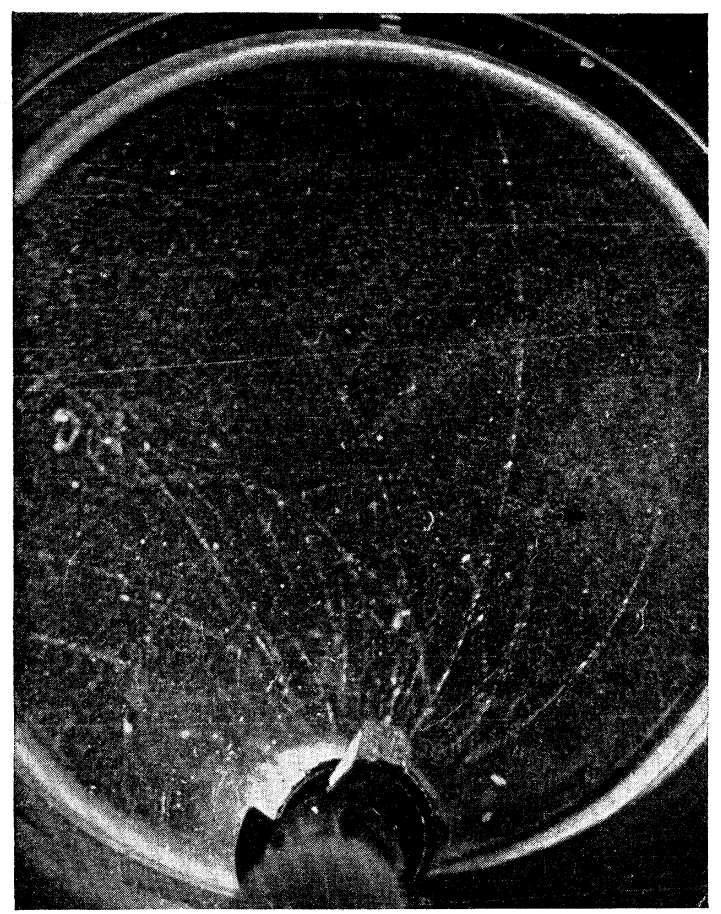

FIG. 2. Electrons from the disintegration of $\mathrm{Li}^{8}$. Magnetic Field $=1500$ gauss.

various spectra. Of primary importance is the scattering of the particles by the gas and vapor in the chamber. Single scatterings greater than one or two degrees can be detected and tracks so scattered must in most cases be rejected. Since scattering is inversely proportional to the square of the energy of the particle, a greater fraction of the low energy tracks is rejected. Scatterings less than one degree are in general undetectable and cause a spread in the distribution which is especially troublesome at the high energy end of the spectrum where the actual number of tracks is small. The scattering can be greatly reduced by employing gases of low atomic weight in the chamber. However, this advantage is somewhat offset by the increased difficulty in accurately measuring the less dense tracks secured in the low weight gases.

In high energy spectra a second source of error becomes important. This error arises because it is impractical to diaphragm the electrons emerging from the target so as to restrict electrons of all energies to the same observational solid angle. Since tracks of small curvature can be of measurable length within regions closer to the target than tracks of large curvature, the low energy end of the spectrum is favored. However, only tracks of which the radius of curvature is of the order of the diameter of the chamber or less are so favored.

Since stereoscopic pictures were not taken an error arises from the angle made by the particles with the magnetic field. The actual particle energy is obtained by dividing the measured energy by the sine of the angle with the field. For observed tracks this angle is greater than $80^{\circ}$, introducing a maximum error of about two percent.

Precautions must be taken to shut off the beam completely during the chamber expansion so as to avoid photographing electrons ejected from the target assembly by gamma-rays given off during the bombardment. Contamination effects are troublesome; oxygen and nitrogen occluded in the targets and carbon deposited during bombardment are the worst offenders. Additional errors arise from a small spread in the loss of energy of the particles as they pass through the foil separating target from chamber, from deviations in the magnetic field value, from imperfect reprojection and from human fallibility in measuring tracks.

\section{Analysis of the Data}

The data secured in the manner described above yield the number of tracks corresponding to a given $H \rho$ value where $H$ is the magnetic field employed and $\rho$ is the track radius to within one-half centimeter. Since the momentum of a particle of charge $e$ is given by $P=H \rho e / c$ we secure fundamentally the distribution in momentum of the particles. The observed particles have lost momentum in passing through the foil between target and chamber so that

$$
H \rho=H \rho_{\text {obs }}+\delta(H \rho),
$$

where $\delta(H \rho)$ for a foil of known material and thickness can be calculated from the data given in Chapter XIV of Radiations from Radioactive Substances, by Rutherford, Chadwick and Ellis. The fact that $\delta(H \rho)$ for a given foil decreases with increasing $H \rho$ necessitates a small correction in 
the number of tracks observed within a given $H \rho$ interval in order to secure the correct relative number in that interval.

Since in connection with the production of radioactive nuclei the quantities of interest are the average energy, the most probable energy, and the maximum energy of the emitted particles we have thought it advisable to plot the data as a distribution in energy. This can be done by employing the relations

$$
\begin{gathered}
N(E) \Delta E=N(H \rho) \Delta H \rho, \\
E=m_{0} c^{2}\left[\left(1+\left(H \rho e / m_{0} c^{2}\right)^{2}\right)^{\frac{1}{2}}-1\right] \\
=0.51\left[\left(1+(H \rho / 1700)^{2}\right)^{\frac{1}{2}}-1\right] \mathrm{MEV} ., \\
\Delta E=e^{2} \frac{H \rho}{E+m_{0} c^{2}} \Delta H \rho=\underset{c}{e} \Delta H \rho,
\end{gathered}
$$

where $m_{0}$ is the rest mass, $v$ the velocity, $E$ the energy and $H \rho e / c$ the momentum of the electrons.

\section{Theoretical Formulae}

In order to account for the continuous distribution in energy of the natural beta-particles and to retain the principle of the conservation of energy in the transition between the quantized parent and daughter nuclei, Pauli was led in 1928 to postulate the simultaneous emission with the electron of a particle with zero charge, spin of one-half and small rest mass. The name neutrino has since been given to this particle. The distribution in momentum of the electrons has been calculated by $\mathrm{Fermi}^{3}$ upon certain assumptions as to the coupling energy between the electronneutrino field and the nucleus. For low atomic numbers his result is

$$
N\left(\eta_{e}\right) d \eta_{e}=c \eta_{e}^{2} \eta_{\nu}^{2} d \eta_{e}
$$

where $\eta_{e}=H \rho / 1700$ is the electron momentum in units $m_{0} c, \eta_{\nu}$ is the neutrino momentum in the same units, and $C$ is a factor approximately independent of the momenta for small $z$. A modification by Konopinski and Uhlenbeck ${ }^{4}$ employing

\footnotetext{
${ }^{3}$ Fermi, Zeits. f. Physik 88, 161 (1934).

${ }^{4}$ Konopinski and Uhlenbeck, Phys. Rev. 48, 7, 107 (1935)
}

a different interaction energy yields

$$
N\left(\eta_{e}\right) d \eta_{e}=c \eta_{e}^{2} \eta_{\nu}{ }^{4} d \eta_{e} .
$$

From the theoretical formulae it is possible to determine the ratio of the maximum energy to the average energy of the electron or positron. For large energies compared to $m_{0} c^{2}$ this ratio is found to be 2.0 on the Fermi theory and 2.6 on the Konopinski-Uhlenbeck modification.

A correlation between the observed distributions and the theoretical formulae was attempted by Crane, Delsasso, Fowler and Lauritsen ${ }^{5}$ in the cases of $\mathrm{Li}^{8}$ and $\mathrm{F}^{20}$. Professor J. R. Oppenheimer pointed out that the observed asymmetry of the spectrum was in agreement with the KonopinskiUhlenbeck formula and it was found possible to secure by trial fair agreement with this formula. It has since been pointed out by Kurie, Richardson and Paxton ${ }^{6}$ that the two theoretical formulae can be expressed in the form

$$
\left(N / \eta_{e}^{2}\right)^{1 / \alpha}=k\left(\left(1+\eta^{2}{ }_{\text {max }}\right)^{\frac{1}{2}}-\left(1+\eta_{e}^{2}\right)^{\frac{1}{2}}\right),
$$

where $\alpha=2$ on the Fermi theory and $\alpha=4$ on the Konopinski-Uhlenbeck modification. Thus plots of $\left(N /(H \rho)^{2}\right)^{1 / \alpha}$ against $\left[1+(H \rho / 1700)^{2}\right]^{\frac{1}{2}}$ should be linear for $\alpha=2$ if the Fermi form of the interaction energy is correct and for $\alpha=4$ if the Konopinski-Uhlenbeck form is correct. If such a linear plot is secured extrapolation to the abscissa yields the maximum value of the electron momentum from which the maximum energy can be computed. Such plots have been made for all the data and will be discussed in connection with the separate radioactive elements.

\section{Electron Emitters}

The electron emitters discussed in this paper are distinguished by short half-lives and high energy spectra. The half-lives of $\mathrm{Li}^{8}$ and $\mathrm{B}^{12}$ of 0.5 and 0.02 second, respectively, made it feasible to bombard only for a short time before expansion in order to effect a saving of deuterium. In the case of $\mathrm{B}^{12}$, bombardment actually took place during expansion but runs made with absorbers about the target tube

${ }^{5}$ Crane, Delsasso, Fowler and Lauritsen, Phys. Rev. 47, 971 (1935).

${ }^{6}$ Kurie, Richardson and Paxton, Phys. Rev. 48, 167 (1935). 
showed that the number of electrons ejected by gamma-rays was negligible. In all other cases bombardment was continuous except at the actual time of expansion and film exposure so that an equilibrium state yielding the maximum number of disintegrations per second was reached. Air at a pressure of one atmosphere and ethyl alcohol vapor were employed in the chamber. In order to collimate electrons of the energies found $(\sim 10 \mathrm{MEV})$ it would have been necessary to employ lead diaphragms up to one centimeter in thickness with consequent large probability of scattering from the faces of the aperture. For this reason an aperture as large as the target was cut in the side of the holder facing the chamber. This aperture was covered by a copper foil of 0.6 MEV stopping power in the case of $\mathrm{B}^{12}$ and of $0.3 \mathrm{MEV}$ stopping power in the cases of $\mathrm{Li}^{8}$ and $\mathrm{F}^{20}$. The solid angle of observation was determined by the illumination of the chamber and was of the order of $4 \pi / 25$. The use of air in the chamber and the use of large aperture windows, although acting in opposite directions in distorting the distribution in energy, make the spectra secured uncertain in the low energy regions. Magnetic fields of 1500 gauss were employed, the maximum radius of curvature of the tracks in this field being 30 centimeters.

\section{Positron Emitters}

The positron emitters discussed in this paper are characterized by spectra having maximum energies of the order of $2 \mathrm{MEV}$ or less. The targets were placed in a cylindrical brass holder of $3 / 32$ inch wall thickness, this thickness being sufficient to stop electrons of energies up to 3 MEV. Positrons of all energies were restricted to the same observational solid angle by a window $1 / 16$ inch wide in the wall of the holder. The solid angle was of the order of $4 \pi / 100$. One-mil copper foil was soldered over the window. The correction for average loss of $H \rho$ in such a foil is 100 gauss-centimeters for millionvolt electrons and 230 gauss-centimeters for 100-kilovolt electrons. The low energies of the positrons also necessitated the use of a gas of low scattering power in the chamber. Helium was employed at $100 \mathrm{~cm}$ pressure with ethyl alcohol as the vapor. Helium was preferred to hydrogen because of its larger viscosity and a consequent slower fall of the tracks within it. Magnetic fields of 540 gauss were used, the maximum radius of curvature of the tracks being $15 \mathrm{~cm}$.

In the following paragraphs the spectra and half-lives of $\mathrm{Li}^{8}, \mathrm{~B}^{12}, \mathrm{C}^{11}, \mathrm{~N}^{13}, \mathrm{~N}^{16}, \mathrm{O}^{15}$ and $\mathrm{F}^{20}$ will be discussed in the order of their investigation in this laboratory. In addition, work done on the products accompanying the formation of these elements with special reference to the energy balances of the reactions involved will be presented.

$\mathrm{B}^{12}$

Electrons from the disintegration of boron by deuterons were first reported from this laboratory. ${ }^{7}$ From the spectrum of 1773 tracks taken from 1000 photographs the maximum energy was estimated to be $11 \mathrm{MEV}$ and the average energy to be $4.5 \mathrm{MEV}$. From a count of the number of tracks observed for chamber expansions occurring at different times after bombardment, the half-life of $\mathrm{B}^{12}$ was found to be approximately 0.02 second.

A plot of the data for comparison with the Fermi theory and the Konopinski-Uhlenbeck modification is shown in Fig. 3. The KonopinskiUhlenbeck plot is seen to be linear whereas the Fermi plot is definitely curved. Due to the uncertainty in the spectrum below $3 \mathrm{MEV}$ the agreement with the Konopinski-Uhlenbeck theory in this region may be fortuitous. It is felt, however, that the remainder of the spectrum is sufficiently reliable to indicate a serious disagreement with the predictions of the Fermi theory. Extrapolation of the linear curve to the abscissa yields $\left(1+\eta^{2}{ }_{\text {max }}\right)^{\frac{1}{2}}=26.6$ so that $E^{-}{ }_{\max }=$ 13.0 MEV. This value is $1.5 \mathrm{MEV}$ higher than the energy of any observed track.

In the original letter it was proposed that the $\mathrm{B}^{12}$ was formed in the reaction

$$
{ }_{5} \mathrm{~B}^{11}+{ }_{1} \mathrm{H}^{2} \rightarrow{ }_{5} \mathrm{~B}^{12}+{ }_{1} \mathrm{H}^{1}+Q_{1}
$$

and that it disintegrated according to

$$
{ }_{5} \mathrm{~B}^{12} \rightarrow{ }_{6} \mathrm{C}^{12}+e^{-}-e_{0}^{-}+\nu+Q_{2} .
$$

${ }^{7}$ Crane, Delsasso, Fowler and Lauritsen, Phys. Rev. 47, 887 (1935). 


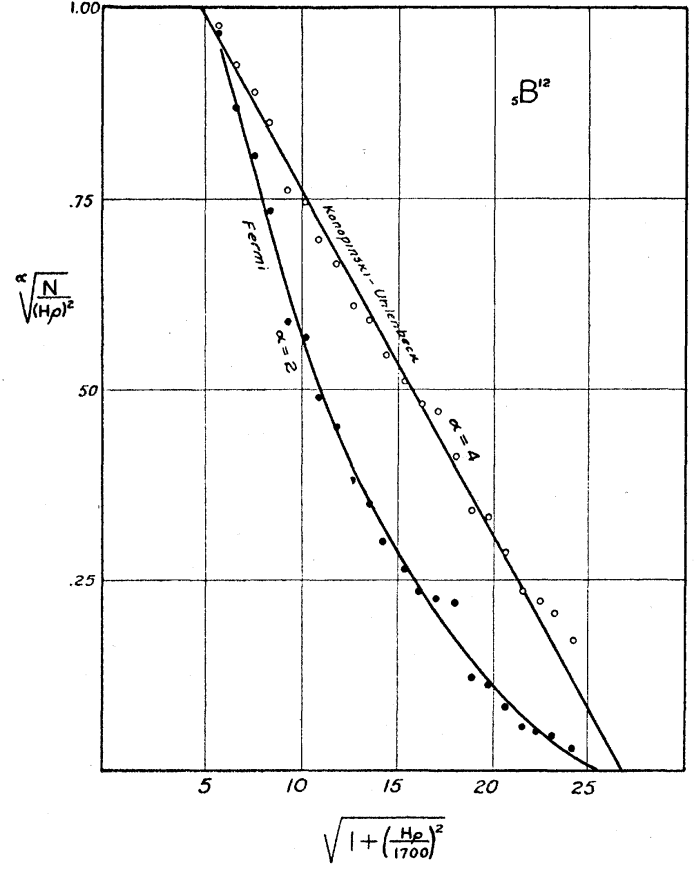

FIG. 3. Fermi and Konopinski-Uhlenbeck plots for the electrons from $\mathrm{B}^{12}$.

By bombarding the target during the chamber expansion and by employing foils of approximately $65-\mathrm{cm}$ stopping power, it was possible simultaneously to observe electrons and the longest range group $(92 \mathrm{~cm})$ of the protons first reported by Cockcroft and Walton ${ }^{8}$ from the disintegration of boron by deuterons. Approximately 20 electrons per proton were observed and hence it was pointed out that these protons could not accompany the formation of $\mathrm{B}^{12}$. Since two other proton groups at $58 \mathrm{~cm}$ and $30 \mathrm{~cm}$ were reported by the same observers to be of approximately the same intensity as the 92 $\mathrm{cm}$ group, they were ruled out on similar grounds. A much more intense group at $9 \pm 2 \mathrm{~cm}$ was taken as determining an upper limit for $Q_{1}$. Some evidence has since been found in this laboratory which would indicate that even these low energy protons are not produced in quantities equal to the disintegration electrons. In any case $Q_{1} \leq 2.5 \mathrm{MEV}$.

The two reactions given above were compared with

$$
{ }_{5} \mathrm{~B}^{11}+{ }_{1} \mathrm{H}^{2} \rightarrow{ }_{6} \mathrm{C}^{12}+{ }_{0} n^{1}+Q_{3},
$$

${ }^{8}$ Cockcroft and Walton, Proc. Roy. Soc. A144, 704 (1934) where $Q_{3}$ was taken as approximately equal to $13 \mathrm{MEV}$ in accordance with unpublished measurements made by Bonner and Brubaker. The masses proposed by Bethe ${ }^{9}$ yield $Q_{3}=12.2$ $\pm 1.3 \mathrm{MEV}$. Upon calculation of $Q_{2}$ it was found that

$$
{ }_{5} \mathrm{~B}^{12}{ }_{6} \mathrm{C}^{12}+11 \mathrm{MEV}
$$

and upon comparison with the observed values of $E^{-}$max $\sim 11 \mathrm{MEV}$ and $E^{-}$av $\sim 4.5 \mathrm{MEV}$ it was concluded that the energy lost by $\mathrm{B}^{12}$ in disintegrating into $\mathrm{C}^{12}$ corresponded to the upper limit of energy of the electron spectrum. The large energy involved in the electron spectrum and the fact that only the mass difference between the neutron and the proton was made use of placed this conclusion well beyond the limits of experimental error, provided only that the reactions assumed were correct. A similar conclusion had been reached by Henderson ${ }^{10}$ from a very precise but less direct experiment, namely, a comparison of the change of energy around the two branches of the Th C to Th D sequence. As will be seen later, this result is important in connection with the energy consideration of reactions involving the production of positron emitters where more accurate experimental data are available. It will be assumed in the subsequent material that the difference in mass of a radioactive element and the resulting product is given by

$$
c^{2} \Delta m=E^{ \pm}{ }_{\max }+e^{ \pm} \pm e_{0}^{-}+\nu .
$$

In our previous notation we can therefore write $Q_{2}=E^{-}{ }_{\text {max }}$ and $T_{2}=E^{+}{ }_{\text {max }}$. Evidence for a very small or zero neutrino mass will be presented later.

$\mathrm{Li}^{8}$

In a Letter to the Editor of the Physical Review, Crane, Delsasso, Fowler and Lauritsen ${ }^{5}$ showed the distribution in energy of 1646 electrons obtained from 1000 cloud chamber photographs of tracks obtained by bombarding a lithium chloride target with deuterons. It was shown that the major portion of the spectrum could be represented by the KonopinskiUhlenbeck formula if the maximum energy was assumed to be slightly greater than the end point given by inspection at $10 \mathrm{MEV}$. The agreement

${ }^{9}$ Bethe, Phys. Rev. 47, 633 (1935).

${ }^{10}$ Henderson, Proc. Roy. Soc. A147, 572 (1934). 
between the observed and theoretical distribution is clearly shown in Fig. 4. From this plot the maximum energy is found to be $11.2 \mathrm{MEV}$.

The formation of $\mathrm{Li}^{8}$ was attributed to the transmutation of $\mathrm{Li}^{7}$, the reaction being

$$
{ }_{3} \mathrm{Li}^{7}+{ }_{1} \mathrm{H}^{2} \rightarrow{ }_{3} \mathrm{Li}^{8}+{ }_{1} \mathrm{H}^{1}+Q_{1} .
$$

Disintegration of the $\mathrm{Li}^{8}$ was believed to result in the formation of $\mathrm{Be}^{8}$ or of two alpha-particles according to

or

$$
{ }_{3} \mathrm{Li}^{8} \rightarrow{ }_{4} \mathrm{Be}^{8}+e^{-}-e_{0}^{-}+\nu+Q_{2}
$$

$$
{ }_{3} \mathrm{Li}^{8} \rightarrow 2{ }_{2} \mathrm{He}^{4}+e^{-}-e_{0}{ }^{-}+\nu+Q_{2}{ }^{\prime} .
$$

Measurement of the half-life of the $\mathrm{Li}^{8}$ by counting the number of tracks observed for chamber expansions occurring at different times after bombardment yielded $\tau=0.5$ second.

The protons believed to accompany the formation of $\mathrm{Li}^{8}$ were reported by the authors in November, 1935. ${ }^{11}$ By observing, in the cloud chamber, protons emitted by a lithium oxide target under deuteron bombardment at $700 \mathrm{kv}$ peak two distinct groups were found at $31.7 \pm 0.5 \mathrm{~cm}$

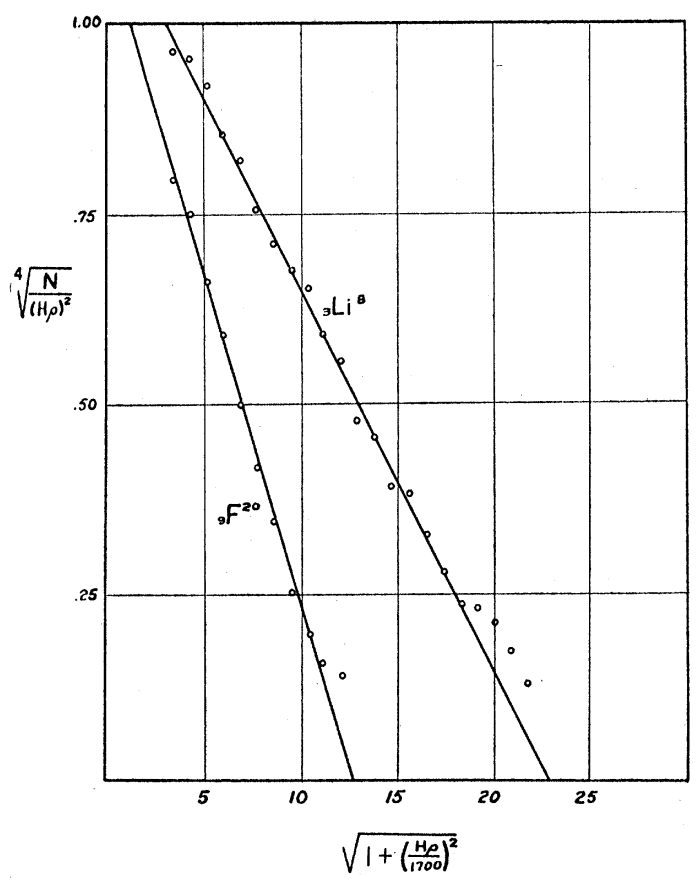

FIG. 4. Konopinski-Uhlenbeck plots for the electrons from $\mathrm{Li}^{8}$ and $\mathrm{F}^{20}$.

${ }^{11}$ Delsasso, Fowler and Lauritsen, Phys. Rev. 48, 848 (1935). and $26 \pm 1 \mathrm{~cm}$. The longer range protons were first reported by Cockcroft and Walton ${ }^{8}$ and were shown by Oliphant, Shire and Crowther ${ }^{12}$ to be due to the transmutation of $\mathrm{Li}^{6}$ by deuterons into $\mathrm{Li}^{7}$. The $26-\mathrm{cm}$ particles were equal in number to about one-fifth the number of the longer range particles and were found to correspond to the number of electrons from $\mathrm{Li}^{8}$ within a factor of two or three. Their energy was given as $4.3 \pm 0.1 \mathrm{MEV}$ yielding $Q_{1}=4.3$ $\pm 0.1 \mathrm{MEV}$. Upon comparison with

$$
{ }_{3} \mathrm{Li}^{7}+{ }_{1} \mathrm{H}^{2} \rightarrow{ }_{4} \mathrm{Be}^{8}+{ }_{0} n^{1}+Q_{3},
$$

where $Q_{3}=14.3 \pm 0.5 \mathrm{MEV}$ from measurements made by Bonner and Brubaker,* we can calculate that $Q_{2}=10.5 \pm 0.6 \mathrm{MEV}$ which corresponds closely to the maximum energy of the electron spectrum.

\section{$\mathrm{F}^{20}$}

Upon bombardment by fast deuterons a calcium fluoride target was found to emit electrons of approximately one-half the maximum energy found in the cases of lithium and boron. The spectrum of 1363 tracks obtained from 800 cloud chamber photographs has been published previously. ${ }^{5}$ It was found possible to adjust the Konopinski-Uhlenbeck curve with end point at 5.2 MEV to the observed data although the end point by inspection allowing for scattering seemed to be at approximately $4.8 \mathrm{MEV}$. A graph of the data for comparison with the Konopinski-Uhlenbeck theory is shown in Fig. 4. Four tracks of much greater energy than the remainder of the tracks have been omitted as they were thought to be due to minute contamination of the target holder by lithium or boron. From this plot we find that $E^{-}$max $=5.9 \mathrm{MEV}$. Using an ionization chamber we found the halflife to be about 12 seconds.

In looking for the protons accompanying the formation of $\mathrm{F}^{20}$ we have observed two groups of particles with ranges at 15.5 and $10.0 \mathrm{~cm}$ from calcium fluoride under deuteron bombardment at $900 \mathrm{kv}$. The group at $15.5 \mathrm{~cm}$ is most certainly due to carbon or deuterium deposited on the target during bombardment, while the group

${ }^{12}$ Oliphant, Shire and Crowther, Proc. Roy. Soc. A146, 922 (1934).

* Bonner and Brubaker, Phys. Rev. 47, 973 (1935). 
at $10.0 \mathrm{~cm}$ could be due to oxygen adsorbed on the target. However, protons of this last range have not been observed in the same quantities from other targets under deuteron bombardment. These protons have energy $2.5 \pm 0.2 \mathrm{MEV}$ so that if they accompany the formation of $\mathrm{F}^{20}$ in the reaction

$$
{ }_{9} \mathrm{~F}^{19}+{ }_{1} \mathrm{H}^{2} \rightarrow{ }_{9} \mathrm{~F}^{20}+{ }_{1} \mathrm{H}^{1}+Q_{1}
$$

we have $Q_{1}=1.7 \mathrm{MEV}$. Using this value and $\mathrm{F}^{19}=19.0041^{13}$ we find $\mathrm{F}^{20}=20.0084$. The disintegration of $\mathrm{F}^{20}$ results in the production of $\mathrm{Ne}^{20}$ according to

$$
{ }_{9} \mathrm{~F}^{20} \rightarrow{ }_{10} \mathrm{Ne}^{20}+e^{-}-e_{0}^{-}+\nu+Q_{2},
$$

from which the mass of $\mathrm{Ne}^{20}$ is calculated to be 20.0021. This value is approximately $3 \mathrm{MEV}$ higher than the value given by Bainbridg $\mathrm{e}^{14}$ when corrected to the scale of masses proposed by Bethe.

\section{$\mathrm{N}^{13}$}

Since the discovery of $\mathrm{N}^{13}$, the first artificially produced radioactive element, numerous attempts have been made to measure its half-life and to determine the maximum energy of the positrons which it emits. Recent measurements by Allison ${ }^{15}$ at Cambridge give the half-life as $10.3 \pm 0.3$ minutes in agreement with the original value 10.3 minutes given by Crane and Lauritsen..$^{16}$

In order to secure accurately the shape of the spectrum and to get a fair estimate of the maxi-

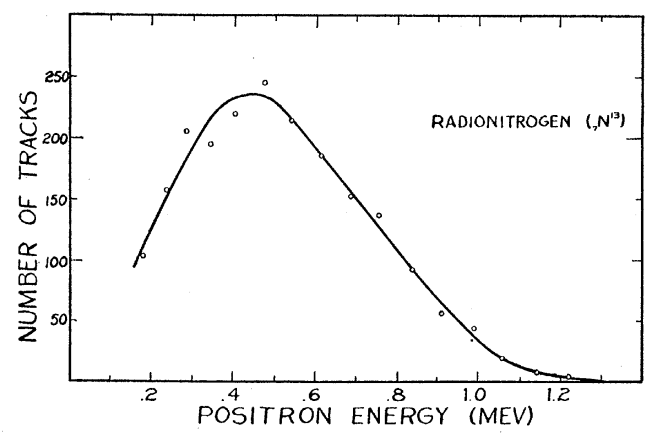

FIG. 5. The distribution in energy of the positrons from $\mathrm{N}^{13}$.

${ }^{13}$ Henderson, Livingston and Lawrence, Phys. Rev. 46, 38 (1934).

${ }_{14}$ Bainbridge, Phys. Rev. 43, 424 (1933).

${ }^{15}$ Allison, Bull. Am. Phys. Soc. 10, 20 (1935)

${ }^{16}$ Crane and Lauritsen, Phys. Rev. 45, 430 (1934).

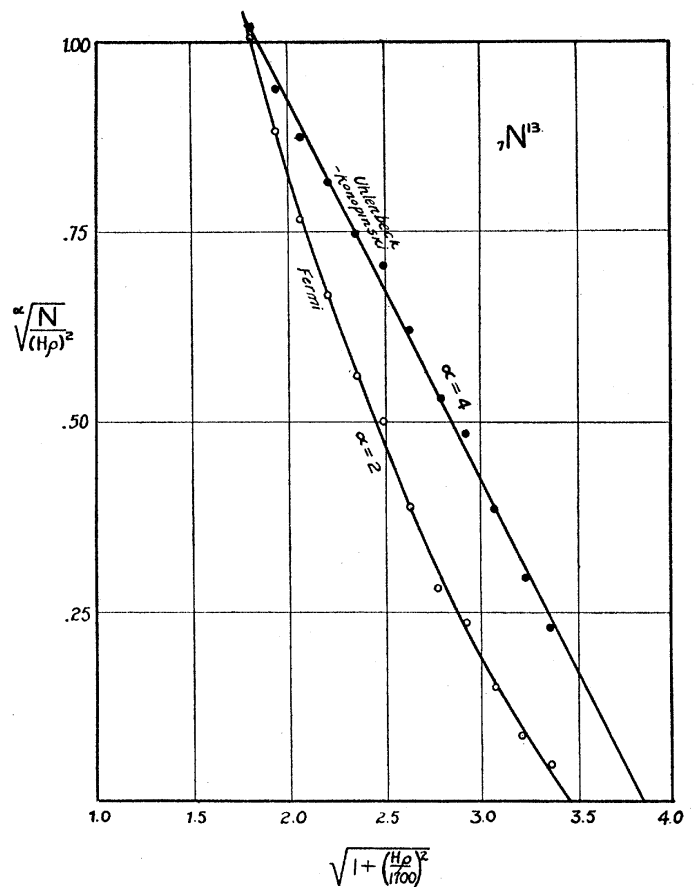

FIG. 6. Fermi and Konopinski-Uhlenbeck plots for the positrons from $\mathrm{N}^{13}$.

mum energy we were prompted to eliminate as far as possible scattering and solid angle distortions in the manner described previously. The distribution in energy of 1762 tracks from 3000 photographs secured by bombarding carbon with $700-\mathrm{kv}$ deuterons is shown in Fig. 5. By inspection, the maximum energy appears to be 1.25 MEV. The Konopinski-Uhlenbeck plot in Fig. 6 is seen to be linear while the Fermi plot is definitely curved. The maximum energy from the Konopinski-Uhlenbeck plot is $1.45 \pm 0.1$ MEV. A similar linear plot and the same maximum energy has been secured by Kurie, Richardson and Paxton at Berkeley. ${ }^{17}$

An analysis of the effect of errors in measurement casts some doubt upon the significance of the apparent agreement of the KonopinskiUhlenbeck formula with experiment. If the upper portion of the $\mathrm{N}^{13}$ spectrum were actually a linear curve extending from 0.5 to $1.1 \mathrm{MEV}$ as one might be led to believe from the form of the curve, then the tail of the curve could be ascribed to observational error. A curve of the form

${ }^{17}$ Kurie, Richardson and Paxton, Phys. Rev. 49, 368 (1936). 


$$
N(x)=k\left(E_{\max }-E\right)=-k x
$$

can be shown to be transformed by Gaussian errors with probable error $d=0.477 / h^{\frac{1}{2}}$ into a curve with the expression

$$
N(x)=\frac{k}{2(\pi h)^{\frac{1}{2}}} e^{-h x^{2}}-\frac{k x}{z}\left[1-\frac{2}{\pi^{\frac{1}{2}}} \int_{0}^{x h^{\frac{1}{2}}} e^{-x^{2}} d x\right] .
$$

Employing this equation we have found that a probable error or deviation of six percent would account completely for the observed tail of the curve. It is felt that deviations of the magnetic field, scattering, and other errors yield a spread somewhat smaller than this, but certainly a number of the tracks with energy greater than 1.1 MEV can be accounted for in this way. There still remains then some uncertainty in determining the value of the maximum energy from the observed distribution in energy.

This problem can of course be solved by an investigation of the energy changes in the reactions describing the modes of disintegration of carbon by deuterons. The reactions are

$$
\begin{aligned}
{ }_{6} \mathrm{C}^{12}+{ }_{1} \mathrm{H}^{2} \rightarrow{ }_{7} \mathrm{~N}^{13}+{ }_{0} n^{1}+T_{1}, \\
{ }_{7} \mathrm{~N}^{13} \rightarrow{ }_{6} \mathrm{C}^{13}+e^{+}+e_{0}+\nu+T_{2}, \\
{ }_{6} \mathrm{C}^{12}+{ }_{1} \mathrm{H}^{2} \rightarrow{ }_{6} \mathrm{C}^{13}+{ }_{1} \mathrm{H}^{1}+T_{3} .
\end{aligned}
$$

The above reactions have been discussed by Tuve and Hafstad, ${ }^{18}$ by Newson, ${ }^{19}$ by Bonner, Delsasso, Fowler and Lauritsen, ${ }^{20}$ and by Kurie, Richardson and Paxton. ${ }^{17}$

Since the bombarding potential enters into the experimental determination of $T_{1}$ and $T_{3}$ we have thought it desirable to measure the energy of the protons and neutrons produced by incident deuterons of the same energy under identical experimental conditions. Employing a cloud chamber filled with methane the energy of the recoil protons making an angle of not more than $16^{\circ}$ with neutrons produced at right angles to $880-\mathrm{kv}$ peak deuterons has been found by Bonner and Brubaker ${ }^{21}$ to extend from 0.10 to $0.35 \mathrm{MEV}$. With these data they calculate $T_{1}$ to be -0.37 $\pm 0.03 \mathrm{MEV}$. We have measured the residual range of protons ejected from a carbon target at $90^{\circ} \pm 5^{\circ}$ with the incident deuteron beam. The

\footnotetext{
18 Tuve and Hafstad, Phys. Rev. 48, 106 (1935).

19 Newson, Phys. Rev. 48, 790 (1935).

20 Bonner, Delsasso, Fowler and Lauritsen, Phys. Rev. 49, 203 (1936).

${ }^{21}$ Bonner and Brubaker (unpublished).
}

protons passed through a copper foil of approximately $5 \mathrm{~cm}$ of air stopping power $\left(9.6 \mathrm{mg} / \mathrm{cm}^{2}\right)$ into an air-filled cloud chamber employing ethyl alcohol as a vapor and operating at an expanded pressure of $116 \mathrm{~cm}$ of mercury at $28^{\circ} \mathrm{C}$. The mean stopping power was taken to be $1.50 \pm 0.05$ from the range of polonium alpha-particles observed under the same conditions. The range of the wellknown alpha-particles from the reaction

$$
{ }_{3} \mathrm{Li}^{7}+{ }_{1} \mathrm{H}^{1} \rightarrow 2{ }_{2} \mathrm{He}^{4}+Q
$$

was used for calibrating the foil. With the value $Q=17.06 \pm 0.06 \mathrm{MEV}$ given by Oliphant, Kempton and Rutherford ${ }^{22}$ it was calculated that these alpha-particles should have an extrapolated range of $8.8 \mathrm{~cm}$ when produced by $650-\mathrm{kv}$ protons. From the data of $\mathrm{Mano}^{23}$ corrections were made for the variation in stopping power of the foil with the velocity of the particles. The abscissa in Fig. 7 represents range in centimeters of air under normal conditions of pressure and temperature. The distributions in range of both the alpha-particles from lithium bombarded by $650-\mathrm{kv}$ peak protons and from carbon bombarded by $600-\mathrm{kv}$ peak deuterons are shown, the greater sensitivity of proton ranges to energy contributed by the bombarding particles being clearly evident. Examples of the tracks from carbon are

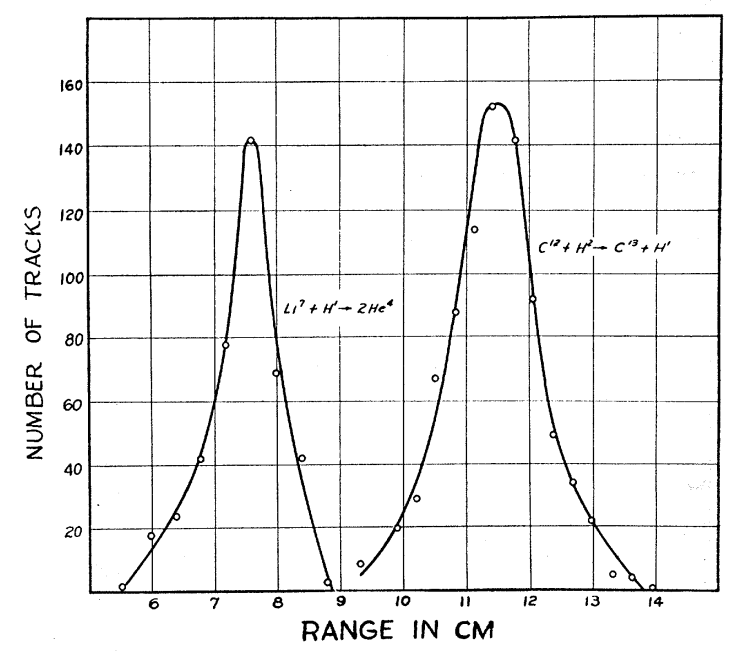

FIG. 7. The distribution in range $\left(760 \mathrm{~mm}, 15^{\circ} \mathrm{C}\right)$ of the protons from carbon bombarded by $600-\mathrm{kv}$ peak deuterons and of the alpha-particles from lithium bombarded by $650-\mathrm{kv}$ peak protons.

${ }^{22}$ Oliphant, Kempton and Rutherford, Proc. Roy. Soc. A149, 406 (1935).

${ }^{23}$ Mano, J. de phys. et rad. 5, 628 (1934). 
TABLE I. Mean range and energy of protons produced by bombarding carbon by deuterons.

\begin{tabular}{cll}
\hline \hline $\begin{array}{c}\text { Peak Bombarding } \\
\text { Potential }\left(E_{d}\right)\end{array}$ & $\begin{array}{c}\text { Mean Range } \\
\text { Protons }\end{array}$ & \multicolumn{1}{c}{ Energy $\left(E_{p}\right)$} \\
\hline $600 \mathrm{kv}$ & $13.5 \mathrm{~cm}$ & $2.96 \pm 0.06 \mathrm{MEV}$ \\
750 & 14.8 & $3.12 \pm 0.15$ \\
900 & 16.4 & $3.32 \pm 0.15$ \\
\hline \hline
\end{tabular}

shown in Fig. 8. The maximum range intercept was taken as corresponding most closely to the extrapolated range of the particles produced at $85^{\circ}$ with bombarding ions corresponding to the peak voltages. The proton straggling was taken as approximately $3 \mathrm{~mm}$ and in Table I, again using data from Mano, we give the mean range and energy of the protons produced by deuterons at various peak voltages.

The data are consistent within the experimental error and using the formula

$$
T_{3}=(1 / 13)\left[14 E_{p}-11 E_{d}-2\left(2 E_{p} E_{d}\right)^{\frac{1}{2}} \cos \theta\right],
$$

where $\theta=85^{\circ}$ we find $T_{3}=2.65 \pm 0.07 \mathrm{MEV}$ in agreement with the value $T_{3}=2.7 \pm 0.1 \mathrm{MEV}$ previously reported by Cockcroft and Walton. ${ }^{8}$ The value $2.65 \mathrm{MEV}$ has also been given by Livingston and Lawrence. ${ }^{19}$

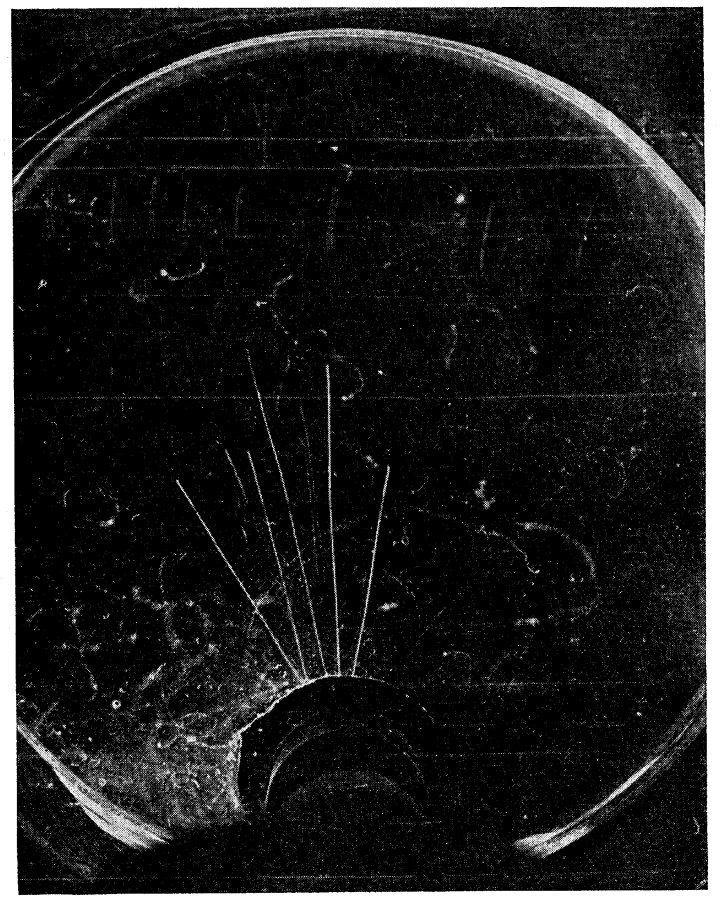

FIG. 8. Protons from the disintegration of $\mathrm{C}^{12}$ by $600-\mathrm{kv}$ deuterons after passage through a foil of $4.8-\mathrm{cm}$ stopping power. Stopping power of air and vapor in chamber $=1.5$.
It will be noted that the assumption has been made that no gamma-rays are emitted in connection with either the observed protons or neutrons. Gamma-rays from carbon bombarded by deuterons have been reported by Crane and Lauritsen $^{24}$ and by Tuve and Hafstad. ${ }^{18}$ The latter observers give the yield as one quantum per $10^{6}$ incident deuterons at $950 \mathrm{kv}$ and the energy of the gamma-rays as $3.7 \mathrm{MEV}$. In this laboratory the same energy was found but the efficiency of production was much lower. Crane and Lauritsen originally found 3 quanta per $10^{8}$ deuterons at $900-\mathrm{kv}$ peak and 3 quanta per $10^{9}$ deuterons at 700-kv peak. Recent estimates from cloud chamber measurements yield values slightly lower than these. Tuve and Hafstad find a yield of 3 protons per $10^{6}$ deuterons at $950 \mathrm{kv}$, we find 2 per $10^{7}$ at $650-\mathrm{kv}$ peak and Cockcroft and Walton report 2 per $10^{8}$ at $500 \mathrm{kv} .^{8}$ Allison ${ }^{15}$ gives the efficiency for production of $\mathrm{N}^{13}$ at $520 \mathrm{kv}$ as 4 per $10^{9}$ deuterons. Definite exclusion of gamma-rays from the reactions of interest is impossible on the above evidence, but it will be evident from the following discussion that the disintegration energies of the reactions can be made consistent without introducing gamma-rays of the observed energy.

The difference in the masses of the neutron and the hydrogen atom cannot be directly measured but is determinable from the disintegration of the deuteron by gamma-rays. The most recent work, that of Feather, ${ }^{25}$ yields a packing energy of 2.26 MEV for the deuteron. Using Bainbridge' ${ }^{26}$ masses for deuterium and hydrogen as adjusted by Bethe to fit $\mathrm{He}^{4}=4.00336$ as standard we find ${ }_{0} n^{1}-{ }_{1} \mathrm{H}^{1}=0.00051=0.48 \mathrm{MEV}$. Recalling formula II (d) and assuming a zero neutrino mass, we find $E^{+} \max =1.52 \mathrm{MEV}$. Bonner and Brubaker ${ }^{27}$ have recently given values of the mass of the deuteron from disintegration data which yields ${ }_{0} n^{1}-{ }_{1} \mathrm{H}^{1}=0.00078$ $=0.73 \mathrm{MEV}$ and $E^{+}{ }_{\max }=1.27 \mathrm{MEV}$. We thus $^{*}$ have two values of the maximum energy of the

\footnotetext{
${ }^{24}$ Crane and Lauritsen, Phys. Rev. 45, 435 (1934).

${ }^{25}$ Feather, Nature 136, 467 (1935).

${ }^{26}$ Bainbridge, Phys. Rev. 43, 103; 44, 56 (1933).

${ }^{27}$ Bonrer and Brubaker, Phys. Rev. 49, 19 (1936).

* Note added in proof. The mass spectrographic values for hydrogen (1.00812) and deuterium (2.01471) given recently by Aston, Nature 137, 357 (1936) yield ${ }_{0} n^{1}-{ }_{1} \mathrm{H}^{1}=0.00089$ $=0.83 \mathrm{MEV}$ and a still lower maximum value for the positron spectrum, namely $1.22 \mathrm{MEV}$.
} 


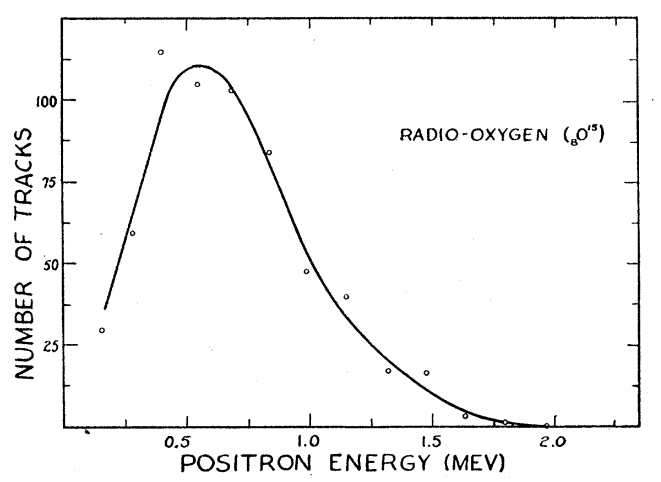

FIG. 9. The distribution in energy of the positrons from $\mathrm{O}^{15}$.

positron from the energy balance of the double reactions between which it is difficult to decide. On the other hand, the maximum energy from the observed spectrum is by inspection equal to 1.25 MEV and on the Konopinski-Uhlenbeck extrapolation to $1.45 \mathrm{MEV}$. A decision between these values seems to us to be at present impossible. In any case, the discrepancy is of the order of $250 \mathrm{kv}$ so that it can be estimated that the neutrino mass is less than one-half the electron mass.

$\mathrm{O}^{15}$

Radioactive $\mathrm{O}^{15}$ was first produced by McMillan and Livingston ${ }^{28}$ who found the half-life to be $126 \pm 5$ seconds. We have bombarded a fused target of sodium nitrite with $900-\mathrm{kv}$ peak deuterons and obtained a total of 528 tracks from 1800 photographs. The distribution in energy of these positrons is shown in Fig. 9 and the Konopinski-Uhlenbeck plot in Fig. 10. We find by inspection $E^{+}{ }_{\max }=1.7 \mathrm{MEV}$ and from the Konopinski-Uhlenbeck extrapolation $E^{+}{ }_{\max }=2.0$ $\mathrm{MEV}$. The positrons were attributed to

$$
\begin{aligned}
{ }_{7} \mathrm{~N}^{14}+{ }_{1} \mathrm{H}^{2} \rightarrow{ }_{8} \mathrm{O}^{15}+{ }_{0} n^{1}+T_{1}, \\
{ }_{8} \mathrm{O}^{15} \rightarrow{ }_{7} \mathrm{~N}^{15}+e^{+}+e_{0}{ }^{-}+\nu+T_{2},
\end{aligned}
$$

since sodium has not been found to yield positrons under deuteron bombardment and the formation of $\mathrm{F}^{17}$ from oxygen takes place only for bombarding potentials above 2 MEV. ${ }^{19}$ Since $Q_{1}$ has not as yet been determined an energy balance with the reaction

$$
{ }_{7} \mathrm{~N}^{14}+{ }_{1} \mathrm{H}^{2} \rightarrow{ }_{7} \mathrm{~N}^{15}+{ }_{1} \mathrm{H}^{1}+T_{3},
$$

${ }^{28}$ McMillan and Livingston, Phys. Rev. 47, 452 (1935). where $T_{3}=8.0 \pm 0.3 \mathrm{MEV}^{29}$ cannot be attempted and it suffices to point out that the neutrons should have an energy equal to $4.2 \mathrm{MEV}$ for deuterons of zero bombarding potential.

$\mathrm{N}^{16}$

On the photographs obtained in bombarding sodium nitrite about 110 tracks of negative electrons which could be divided into two distinct groups were counted. The first group terminated at approximately $2 \mathrm{MEV}$ and can be attributed to $\mathrm{Na}^{24}$ produced from the sodium in the target while the second group (equal in number to about five percent of the number of positrons) extended up to $6 \mathrm{MEV}$ and is probably due to $\mathrm{N}^{16}$ produced according to

$$
{ }_{7} \mathrm{~N}^{15}+{ }_{1} \mathrm{H}^{2} \rightarrow{ }_{7} \mathrm{~N}^{16}+{ }_{1} \mathrm{H}^{1}+Q_{1}
$$

and disintegrating according to

$$
{ }_{7} \mathrm{~N}^{16} \rightarrow{ }_{8} \mathrm{O}^{16}+e^{-}-e_{0}-+\nu+Q_{2} .
$$

This group has an average energy of $2.5 \mathrm{MEV}$ which yields a maximum energy from the Konopinski-Uhlenbeck theory of $6.5 \mathrm{MEV}$. The half-life of $\mathrm{N}^{16}$ has been determined by Fermi, Amaldi, d'Agostino, Rasetti and Segrè ${ }^{30}$ as approximately 9 seconds, the radioactive substance being produced by the bombardment of fluorine by neutrons with the emission of an alpha-particle. Livingston, Henderson and Lawrence give the half-life as $10.0 \pm 0.5$ seconds. $^{31}$

\section{$\mathrm{C}^{11}$}

The disintegration of boron by deuterons with the production of a positron emitter was reported by Henderson, Livingston and Lawrence ${ }^{32}$ and by Crane and Lauritsen, ${ }^{16}$ the latter observers giving the half-life as approximately 20 minutes. Recent determinations by Allison $^{15}$ yield a half-life of $21.0 \pm 0.6$ minutes.

A target of amorphous boron bombarded by one-half microampere of $900-\mathrm{kv}$ peak deuterons yielded 604 tracks on 1000 photographs. The energy spectrum is shown in Fig. 11 and the

${ }^{29}$ Lawrence, McMillan and Henderson, Phys. Rev. 47, 253 (1935).

${ }^{30}$ Fermi, Amaldi, d'Agostino, Rasetti and Segrè, Proc. Roy. Soc. A196, 483 (1934).

${ }_{31}$ Livingston, Henderson, and Lawrence, Phys. Rev. 46, 325 (1934).

${ }^{32}$ Henderson, Livingston and Lawrence, Phys. Rev. 45, 428 (1934). 


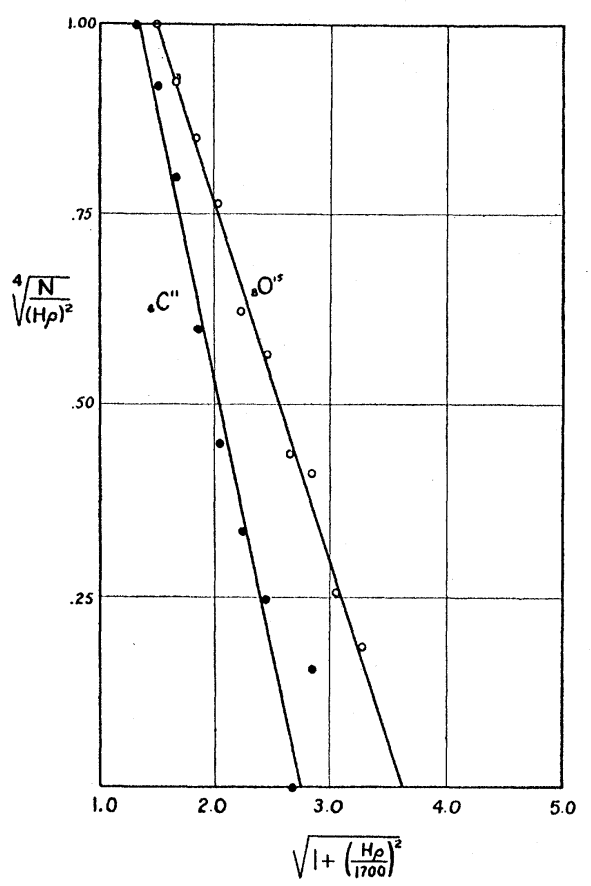

FIG. 10. Konopinski-Uhlenbeck plots for the positrons from $\mathrm{C}^{11}$ and $\mathrm{O}^{15}$.

Konopinski-Uhlenbeck plot in Fig. 10, yielding by inspection $E^{+}{ }_{\max }=1.15 \mathrm{MEV}$ and by extrapolation $E^{+}{ }_{\max }=1.3 \mathrm{MEV}$. The positrons have been attributed to

$$
\begin{aligned}
{ }_{5} \mathrm{~B}^{10}+{ }_{1} \mathrm{H}^{2} & \rightarrow{ }_{6} \mathrm{C}^{11}+{ }_{0} n^{1}+T_{1}, \\
{ }_{6} \mathrm{C}^{11} & \rightarrow{ }_{5} \mathrm{~B}^{11}+e^{+}+e_{0}-+\nu+T_{2} .
\end{aligned}
$$

Because of the deposition of carbon during bombardment and due to the fact that $\mathrm{N}^{13}$ has a greater maximum energy than $\mathrm{C}^{11}$ it was necessary to replace targets frequently. With these considerations in mind, it is not difficult to account for the one or two tracks of energy greater than the Konopinski-Uhlenbeck limit.

\section{$\mathrm{Be}^{10}$ and $\mathrm{C}^{14}$}

The electron emitters described previously do not exhaust the possible nuclear structures containing two more neutrons than protons. Other nuclei of the form ${ }_{z} X^{2 z+2}$ are $\mathrm{Be}^{10}, \mathrm{C}^{14}$ and $\mathrm{O}^{18}$. The last of these has been found to exist to the extent of 0.2 percent in ordinary oxygen ${ }^{33}$ and is believed to be stable. The nucleus $\mathrm{Be}^{10}$ cannot be unstable by more than $0.3 \mathrm{MEV}$

\footnotetext{
${ }^{33}$ Giauque and Johnston, Nature 123, 318 (1929).
}

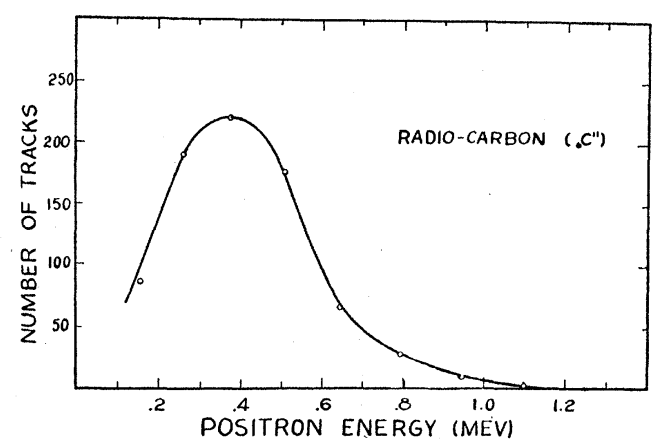

FIG. 11. The distribution in energy of the positrons from $\mathrm{C}^{11}$.

unless it has an exceedingly long half-life or a very small probability of production in the reaction

$$
{ }_{4} \mathrm{Be}^{9}+{ }_{1} \mathrm{H}^{2} \rightarrow{ }_{4} \mathrm{Be}^{10}+{ }_{1} \mathrm{H}^{1}+Q_{1}
$$

since no electron tracks were observed on cloud chamber photographs taken when $\mathrm{Be}^{9}$ was bombarded by $900-\mathrm{kv}$ deuterons.

On the 3000 photographs taken when carbon was bombarded by deuterons four electron tracks between $1 \mathrm{MEV}$ and $5 \mathrm{MEV}$ were observed. These may be attributed to the disintegration of $\mathrm{C}^{14}$ produced according to

$$
{ }_{6} \mathrm{C}^{13}+{ }_{1} \mathrm{H}^{2} \rightarrow{ }_{6} \mathrm{C}^{14}+{ }_{1} \mathrm{H}^{1}+Q_{1},
$$

but are most probably due to lithium or boron contaminations in the target holder. In connection with the determination of the range of the protons from carbon bombarded by deuterons we observed several tracks of energy greater than 4.0 MEV. Dean Wooldridge ${ }^{34}$ of the Norman Bridge Laboratory furnished us with carbon targets prepared from methane in which the proportion of $\mathrm{C}^{13}$ had been increased to 6.6 percent in a diffusion apparatus of the type designed by Hertz. An increase in the percentage of long range tracks was actually found. If these be attributed to the reaction given above then

$$
{ }_{6} \mathrm{C}^{14} \leq{ }_{7} \mathrm{~N}^{14}+1.0 \mathrm{MEV}
$$

but the possibility of contamination must not be overlooked. Fermi, Amaldi, d'Agostino, Rasetti, and Segrè ${ }^{30}$ have bombarded nitrogen with slow neutrons in an attempt to produce $\mathrm{C}^{14}$ according to the reaction

$$
{ }_{7} \mathrm{~N}^{14}+{ }_{0} n^{1} \rightarrow{ }_{6} \mathrm{C}^{14}+{ }_{1} \mathrm{H}^{1},
$$

\footnotetext{
${ }^{34}$ Wooldridge and Jenkins, Phys. Rev. 49, 404 (1936).
} 
but report no activity. Present evidence indicates that $\mathrm{C}^{14}$ is at most slightly unstable with respect to $\mathrm{N}^{14}$.

\section{Discussion of the Results}

From the data presented concerning $\mathrm{Li}^{8}, \mathrm{~B}^{12}$, and $\mathrm{F}^{20}$ it is evident that the KonopinskiUhlenbeck formula is in agreement with the observed distributions in energy of the emitted electrons, although the fact that in the low energy regions of the spectra this agreement may be accidental must not be overlooked. On the other hand, the Fermi distribution is definitely in disagreement with the observed spectra in regions unaffected by the particular experimental arrangements employed.

If the observed positron spectra have not been distorted by errors in measurement exceeding the estimated error then some significance is to be attached to the correspondence with curves of the Konopinski-Uhlenbeck type. Agreement with the Konopinski-Uhlenbeck theory has also been found by Kurie, Richardson and Paxton ${ }^{17}$ in the cases of the electron and positron emitters which they have investigated. The question of the reliability of the maximum energies given by extrapolation of the Konopinski-Uhlenbeck plots can only be answered when a more accurate value of the mass difference between the neutron and the hydrogen atom is available or when experiments for unambiguously determining the end points of the spectra have been devised.

We have incorporated in Table II the numerical results of the investigations outlined above. The maximum energies of the spectra as given by inspection and by the KonopinskiUhlenbeck extrapolation are given. The atomic

TABLE II. Summary of data on the radioactivity of elements of low atomic number.

\begin{tabular}{|c|c|c|c|c|c|c|}
\hline $\begin{array}{l}\text { RADIO- } \\
\text { ACTIVE } \\
\text { ELE- } \\
\text { MENT }\end{array}$ & $\begin{array}{c}\text { PROD- } \\
\text { UCT }\end{array}$ & HALF-LIFE & $\begin{array}{c}E_{\max }( \\
\text { (Insp.) }\end{array}$ & $\begin{array}{c}\text { (MEV) } \\
\text { (K.U.) }\end{array}$ & $\begin{array}{c}E_{\mathrm{av}} \\
(\mathrm{MEV})\end{array}$ & Aтоміс Mass \\
\hline${ }_{5} \mathrm{~B}^{9}$ & ${ }_{4} \mathrm{Be}^{9}$ & - & $0.3^{a}$ & - & - & $9.0140 \pm 0.0010$ \\
\hline${ }_{6} \mathrm{C}^{11}$ & ${ }_{5} \mathrm{~B}^{11}$ & $21 \pm 0.6 \mathrm{~min}$ & 1.15 & 1.3 & 0.43 & $11.0136 \pm .00$ \\
\hline${ }_{7} \mathrm{~N}^{13}$ & ${ }_{6} \mathrm{C}^{13}$ & $10.3 \pm .3 \mathrm{~min}$ & 1.25 & 1.45 & 0.49 & $13.0095 \pm .0008$ \\
\hline${ }_{8} \mathrm{O}^{15}$ & ${ }_{7} \mathrm{~N}^{15}$ & $126 \pm 5$ sec. & 1.7 & 2.0 & 0.62 & $15.0085 \pm .0006$ \\
\hline${ }_{9}^{8} \mathrm{~F}^{17}$ & ${ }_{8}^{1} \mathrm{O}^{17}$ & 70 sec. & $2.1^{\mathrm{b}}$ & $2.4^{\mathrm{b}}$ & & $17.0077 \pm .0003$ \\
\hline${ }_{3} \mathrm{Li}^{8}$ & ${ }_{4} \mathrm{Be}^{8}$ & 0.5 sec. & 10.0 & 11.2 & 3.86 & $8.0185 \pm .000$ \\
\hline${ }_{5} \mathrm{~B}^{12}$ & ${ }_{6} \mathrm{C}^{12}$ & $0.02 \mathrm{sec}$. & 11.0 & 13.0 & 4.75 & $12.0176 \pm .0014$ \\
\hline${ }_{7} \mathrm{~N}^{16}$ & ${ }_{8} \mathrm{O}^{16}$ & $10 \pm .5 \mathrm{sec}$ & 6.0 & 6.5 & 2.5 & $16.0069 \pm .0010$ \\
\hline${ }_{9} \mathrm{~F}^{20}$ & ${ }_{10} \mathrm{Ne}^{20}$ & $12 \mathrm{sec}$. & 5.0 & 5.9 & 2.06 & 20.0084 (?) \\
\hline
\end{tabular}

a Meitner.

b Kurie, Richardson and Paxton. masses of the radioactive elements have been computed by using the Konopinski-Uhlenbeck maximum energies and the masses of the appropriate stable elements as given by Bethe. The half-lives listed are those yielded by the most recent investigations. The average energies of the spectra have been computed in the usual manner from the observed distributions. The data on $\mathrm{F}^{17}$ have been given by Kurie, Richardson and Paxton ${ }^{17}$ and Newson ${ }^{19}$ and on $B^{9}$ by Meitner. ${ }^{35}$

A rapid decrease of the half-lives with increasing maximum energies is indicated by the tabulated results. The Konopinski-Uhlenbeck theory predicts a more rapid decrease than the Fermi theory but too close correlation with the observed results cannot be attempted in view of the dependence of the factor $C$ in the distribution in momentum on a matrix element containing the wave functions of the nucleus which may vary from element to element.

On the neutrino theory of positron decay, the binding energy of the parent nucleus must exceed that of the daughter nucleus by the difference in mass between neutron and proton, plus the rest masses of positron and neutrino, plus the maximum energy of the positron spectrum. If this excess be $\Delta W$, then

$$
\Delta W \sim E^{+}{ }_{\max }+1.5 \mathrm{MEV} .
$$

Now all the positron emitters which we have examined are built up of $p$ protons and $p-1$ neutrons; the nuclei which are formed by their disintegration have $p-1$ protons and $p$ neutrons. The change in binding energy from parent to daughter nucleus is thus to be ascribed entirely to the electrostatic repulsions between protons, if we suppose that all other forces are symmetric between neutron and proton. Since the contribution of this electrostatic repulsion to the energy of a nucleus with $p$ protons is

$$
[p(p-1) / 2] e^{2}(\overline{1 / r}),
$$

where $(\overline{1 / r})$ is the mean of the reciprocal of the distance between protons, we are led to believe that the energy gain from the conversion of a proton to a neutron should be

$$
\left.\Delta W \sim(p-1) e^{2} \overline{1 / r}\right)
$$

${ }^{35}$ Meitner, Nature 22, 420 (1934). 
and thus should increase with atomic number. As is seen from the values in the table, this increase is nearly linear and corresponds to the nearly constant and quite reasonable value

$$
(\overline{1 / r}) \sim 3.5 \times 10^{12} \mathrm{~cm}^{-1} .
$$

The high energy of the electron emitters $\mathrm{Li}^{8}$, $\mathrm{B}^{12}, \mathrm{~N}^{16}$ and $\mathrm{F}^{20}$ can be understood on the basis of their disintegration into the tightly packed nuclei $\mathrm{Be}^{8}, \mathrm{C}^{12}, \mathrm{O}^{16}$, and $\mathrm{Ne}^{20}$. The nuclei $\mathrm{Be}^{10}$, $\mathrm{C}^{14}$, and $\mathrm{O}^{18}$ can be classed together as represent- ing stable or at most slightly unstable configurations.

In conclusion we wish to express our appreciation to Dr. Robert Serber and Dr. J. R. Oppenheimer for discussions of the theoretical aspects of these investigations, to Kurie, Richardson and Paxton for sending us a copy of their manuscript before publication, to Dean Wooldridge for preparation of the carbon targets containing an increased percentage of $\mathrm{C}^{13}$, and to the Seeley W. Mudd fund for financial support.

\title{
A Theoretical Discussion of the Deviation of High Energy Charged Particles in Passing Through Magnetized Iron
}

\author{
W. F. G. Swann, Bartol Research Foundation of the Franklin Institute
}

(Received February 24, 1936)

\begin{abstract}
The paper attempts a critical discussion of the situation pertaining to the magnetic field in a piece of magnetized iron, particularly in relation to the deflecting force which it produces on high speed charged particles passing through it. On the Lorentzian theory the magnetic induction, $B$, is the average value of the true magnetic field $H$, averaged throughout the magnetic material. In this average, regions inside the magnetic entities responsible for the polarization make contributions which determine the whole difference between $B$ and the ordinary macroscopically defined field, $h$, equal to $B-4 \pi I$. A study is made of the special case where the entities are rotating electrically charged spheres.
\end{abstract}

\section{INTRODUCTION}

$T^{1}$ $\mathrm{HE}$ interest of the problem cited in the above title arose, primarily in connection with experiments on the deviation of charged particle cosmic rays in passing through magnetized iron. A simple application of the Lorentzian theory seemed to require that the magnetic induction $B$ was the vector concerned in the deviation while the first experiments performed ${ }^{1}$ seemed to lead to the conclusion that the magnetic intensity $h$ was the vector operative. Subsequent work ${ }^{2}$ has given rise to conclusion that a

${ }^{1}$ B. Rossi, Accad. Lincei, Atti 11, 478 (1930); B. Rossi, Nature 128, 300 (1931); L. M. Mott-Smith, Phys. Rev. 39, 403 (1932).

${ }^{2}$ L. Alvarez, Phys. Rev. 45, 225 (1934); W. F. G. Swann and W. E. Danforth, Phys. Rev. 45, 565 (1934).
If the entities are very small in volume the chance of a point electron missing all of them in its passage through a reasonably small length of the magnetized material is considerable. It appears that for such electrons as miss the entities the effective deflecting force is determined on the average by $h+2 \pi I$. The true average for all electrons passing through the material is determined by $B=h+4 \pi I$; but, that average is contributed to in appreciable amount by very few electrons which experience deflections much in excess of those determined by $B$. These considerations have important consequences in relation to the interpretation of experimental results.

vector being between $B$ and $h$ is the vector really involved. It is not our purpose to discuss here these experiments, or the validity of their interpretation. It will suffice to say that the results to date are such as to suggest that, in such experiments, the elements operative involve features of greater complexity than would be suggested by the most naive view of the matter which regarded the iron as the magnetic equivalent of a bundle of continuous tubes of induction. The purpose of the present paper is to look into certain of the theoretical elements concerned in some degree of detail. In this task we meet at the outset certain difficulties which must be faced.

In the first place while recognizing that the problem should be discussed ultimately in terms 


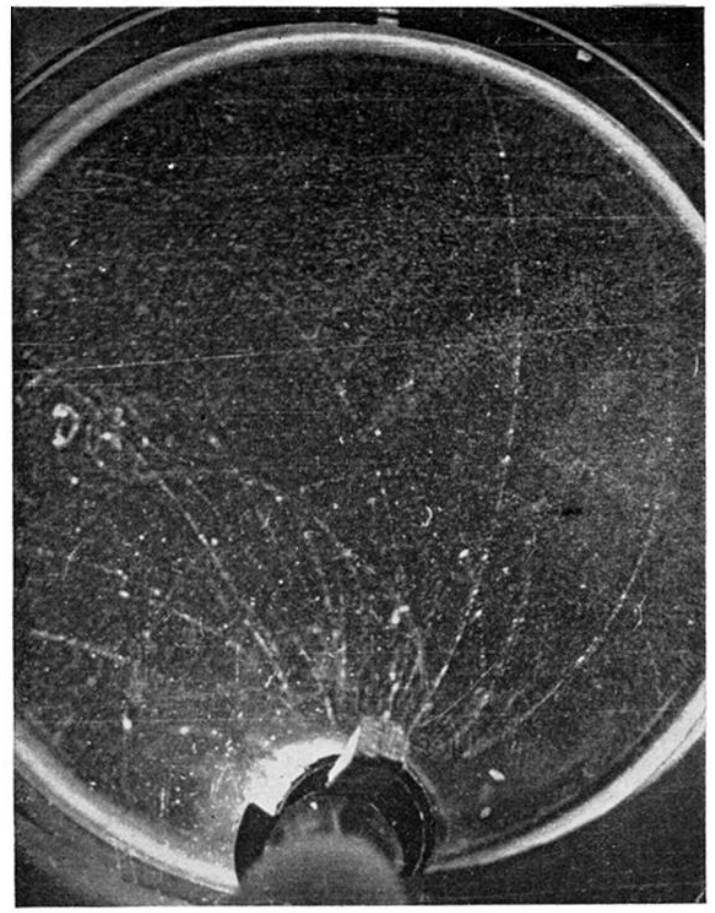

FIG. 2. Electrons from the disintegration of $\mathrm{Li}^{8}$. Magnetic Field $=1500$ gauss. 


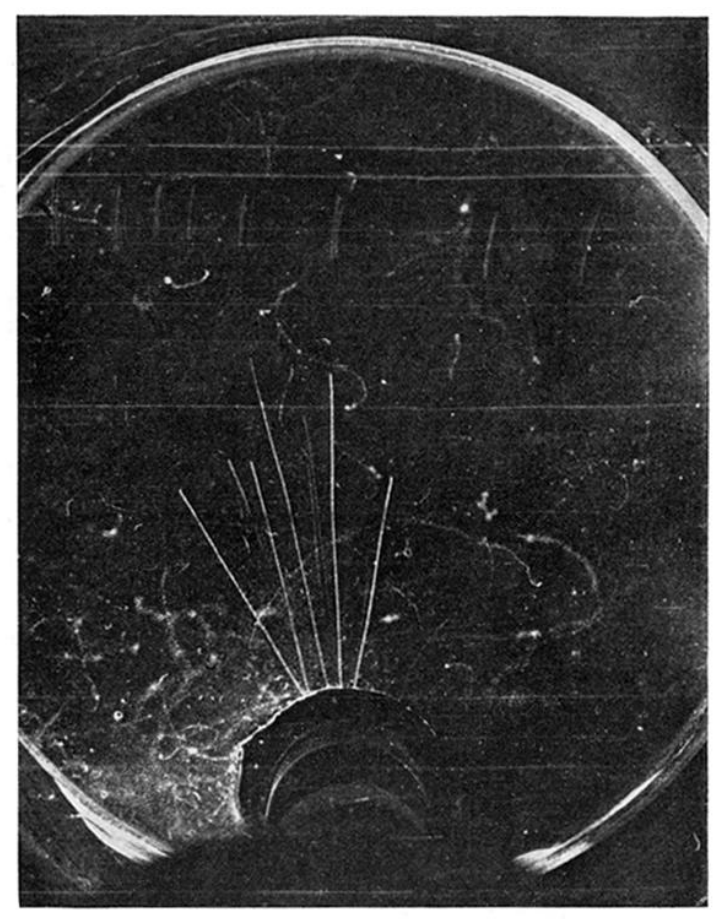

FIG. 8. Protons from the disintegration of $\mathrm{C}^{12}$ by $600-\mathrm{kv}$ deuterons after passage through a foil of $4.8-\mathrm{cm}$ stopping power. Stopping power of air and vapor in chamber $=1.5$. 